\title{
The draft genome sequence of multidrug-resistant Pseudomonas aeruginosa strain CCBH4851, a nosocomial isolate belonging to clone SP (ST277) that is prevalent in Brazil
}

\author{
Melise Silveira $^{1 /+}$, Rodolpho Albano ${ }^{2}$, Marise Asensi' ${ }^{1}$, Ana Paula Carvalho Assef ${ }^{1}$ \\ 'Laboratório de Pesquisa em Infecção Hospitalar, Instituto Oswaldo Cruz-Fiocruz, Rio de Janeiro, RJ, Brasil \\ ${ }^{2}$ Departamento de Bioquímica, Universidade do Estado do Rio de Janeiro, Rio de Janeiro, RJ, Brasil
}

The high occurrence of nosocomial multidrug-resistant (MDR) microorganisms is considered a global health problem. Here, we report the draft genome sequence of a MDR Pseudomonas aeruginosa strain isolated in Brazil that belongs to the endemic clone ST277. The genome encodes important resistance determinant genes and consists of $6.7 \mathrm{Mb}$ with a $\mathrm{G}+\mathrm{C}$ content of $66.86 \%$ and 6,347 predicted coding regions including $60 \mathrm{RNAs}$.

Key words: Pseudomonas aeruginosa - multidrug-resistance - genome sequence

The development of antimicrobial resistance among Gram-negative pathogens has been progressive and relentless. Multidrug-resistant (MDR) Pseudomonas aeruginosa is a pathogen of particular concern (Kanj \& Kanafani 2011).

The presence of a carbapenem-resistant $P$. aerugino$s a$ clone at Brazilian hospitals has been observed in the last two decades (Gales et al. 2003). Strains belonging to this clone, named clone SP, were assigned to ST277 (Fonseca et al. 2010). In Brazil, this clone has been associated with dissemination of the metalo- $\beta$-lactamase SPM-1, which confers broad-spectrum $\beta$-lactam resistance (Gales et al. 2003, Fonseca et al. 2010, Silva et al. 2011). In addition to SPM, ST277 can also carry a class 1 integron (In163) (Carvalho et al. 2006, Fontes et al. 2011) and a $16 \mathrm{~S}$ rRNA methylase ( $r m t \mathrm{D})$ that confers high-level resistance to all 4,6-disubstituted deoxystreptamine aminoglycosides (Doi et al. 2007a, b, Fontes et al. 2011). The accumulation of these resistance determinants imposes an immense limitation on the therapeutic choices available for treatment of $P$. aeruginosa infections.

We present the draft genome sequence of $P$. aeruginosa CCBH4851, a strain isolated in 2008 from the catheter tip of a hospitalised patient in the state of Goiás (Central-West Region of Brazil) and deposited in the Culture Collection of Hospital-Acquired Bacteria (CCBH) located at the Hospital Infection Research Laboratory at Oswaldo Cruz Institute/Oswaldo Cruz Foundation (WDCM947; CGEN022/2010). The strain was resistant to all antimicrobials tested (aztreonam, amikacin, gentamicin, ceftazidime, cefepime, ciprofloxacin, imipenem, mero-

doi: 10.1590/0074-0276140336

Financial support: FIOCRUZ, CNPq

+ Corresponding author: melisechaves@gmail.com

Received 12 September 2014

Accepted 5 November 2014 penem and piperacillin-tazobactam) with the exception of polymyxin B. According to polymerase chain reaction analysis, in addition to In163, this strain has the $b l a_{\text {SPM-1 }}$ and $r m t \mathrm{D}$ genes. Pulsed field gel electrophoresis and multilocus sequence typing analysis also showed that the strain belongs to clone SP and ST277, respectively.

The genomic DNA was sequenced using an Illumina MiSeq sequencing platform. A genomic library was constructed by transposon tagmentation with the Nextera XT DNA Sample Prep kit (Illumina Inc, USA) and sequenced two times with the 300 and 500 cycles MiSeq Reagent v2 kits, which produced 1,445,561 and 1,232,439 paired-end reads, respectively. The data were filtered and trimmed to a Phred score of $>20$ and were assembled de novo using the assembler in Geneious v.6.1.7 (Biomatters Ltd, New Zealand). One-hundred and fifty contigs were generated, with a $\mathrm{G}+\mathrm{C}$ content of $66.86 \%$ and an estimated genome size of $6,719,789 \mathrm{bp}$. Genome annotation was performed on the Rapid Annotation using System Technology (RAST) v.2.0 (available from: rast.nmpdr.org/). In total, 6,347 protein-encoding genes and 60 RNA-encoding genes, 55 tRNAs and 5 rRNAs, were annotated by the RAST server. Among the 568 subsystems identified, the main ones were amino acids and derivatives followed by carbohydrates. Resistance-related genes were analysed using ResFinder 2.1 (acquired antimicrobial resistance gene finder), which is available at the Center for Genomic Epidemiology website (cge.cbs.dtu.dk/services/ResFinder/). Using this approach, we identified resistance genes to aminoglycosides, aacA4 (GenBank JPSS01000146.1, 2,294-2,848 bp), aph(3')IIb (GenBank JPSS01000055.1, 10,770-11,576 bp), aadA7 (GenBank JPSS01000146.1, 3,791-4,588 bp) and rmtD (GenBank JPSS01000080.1, $25,006-25,749 \mathrm{bp}$ ); to $\beta$-lactams, bla $a_{\mathrm{PAO}}$ (GenBank JPSS01000048.1, 31,052-32,245 bp), bla ${ }_{\mathrm{OXA}-50}$ (GenBank JPSS01000001.1, 56,792-57,580 bp), bla ${ }_{\text {OXA-56 }}$ (GenBank JPSS01000146.1, 2,929-3,729 bp) and bla SPM-1 $_{\text {(GenBank }}$ JPSS01000139.1, 1,012-1,842 bp); to fosfomycin, fosA (GenBank JPSS01000029.1, 20,493-20,900 bp); to fenicols, catB7 (GenBank JPSS01000102.1, 3,780-4,418 bp) and $c m x$ (GenBank JPSS01000080.1, 16,868-18,028 bp); 
to sulphonamides, sul1 (GenBank JPSS01000080.1, 19,16320,029 bp; GenBank JPSS01000146.1, 6,468-7,349 bp).

A detailed report of the genomic data analysis, in the context of resistance and virulence characteristics, will be provided in future publications. This announcement of the whole-genome sequencing of the $P$. aeruginosa ST277 strain will provide the basis for elucidating the genetic diversity among clone SP (ST277) strains and allow for further detailed investigations regarding the success of transmission observed for this clone throughout Brazilian states and the tendency of the $b l a_{\mathrm{SPM}-1}$ gene to remain in this clone. Furthermore, the genome sequence will help to understand the specific physiological features of highly successful strains, in general.

This Whole Genome Shotgun project has been deposited in DDBJ/ENA/GenBank under the accession JPSS00000000. The version described in this paper is the first version JPSS01000000.

\section{REFERENCES}

Carvalho AP, Albano RM, de Oliveira DN, Cidade DA, Teixeira LM, Marques EA 2006. Characterization of an epidemic carbapenemresistant Pseudomonas aeruginosa producing SPM-1 metallobeta-lactamase in a hospital located in Rio de Janeiro, Brazil. Microb Drug Resist 12: 103-108.

Doi Y, Garcia DO, Adams J, Paterson DL 2007a. Coproduction of novel $16 \mathrm{~S}$ rRNA methylase RmtD and metallo-beta-lactamase
SPM-1 in a panresistant Pseudomonas aeruginosa insolate from Brazil. Antimicrob Agents Chemother 51: 852-856.

Doi Y, Ghilardi AC, Adams J, Garcia DO, Paterson DL 2007b. High prevalence of metallo-beta-lactamase and 16S rRNA methylase coproduction among imipenem-resistant Pseudomonas aerugino$s a$ isolates in Brazil. Antimicrob Agents Chemother 51: 3388-3390.

Fonseca EL, Freitas FS, Vicente AC 2010. The colistin-only-sensitive Brazilian Pseudomonas aeruginosa clone SP (sequence type 277) is spread worldwide. Antimicrob Agents Chemother 54: 2743.

Fontes LC, Neves PR, Oliveira S, Silva KC, Hachich EM, Sato MI, Lincopan N 2011. Isolation of Pseudomonas aeruginosa coproducing metallo- $\beta$-lactamase SPM-1 and 16S rRNA methylase RmtD1 in an urban river. Antimicrob Agents Chemother 55: 3063-3064.

Gales AC, Menezes LC, Silbert S, Sader HS 2003. Dissemination in distinct Brazilian regions of an epidemic carbapenem-resistant Pseudomonas aeruginosa producing SPM metallo- $\beta$-lactamase. J Antimicrob Chemother 52: 699-702.

Kanj SS, Kanafani ZA 2011. Current concepts in antimicrobial therapy against resistant Gram-negative organisms: extended-spectrum beta-lactamase-producing Enterobacteriaceae, carbapenem-resistant Enterobacteriaceae and multidrug-resistant Pseudomonas aeruginosa. Mayo Clin Proc 86: 250-259.

Silva FM, Carmo MS, Silbert S, Gales AC 2011. SPM-1-producing Pseudomonas aeruginosa: analysis of the ancestor relationship using multilocus sequence typing, 75-pulsed-field gel electrophoresis and automated ribotyping. Microb Drug Resist 17: 215-220. 